VOL. 40 (1989) [381-387]

\title{
STATIONARY SUBSPACES IN ORDERED SPACES
}

\author{
NOBUYUKI KEMOTO
}

\section{Dedicated to Professor Yukihiro Kodama on his 60th birthday.}

\begin{abstract}
In this paper, we shall characterise the $\mathcal{B}(\kappa)$-property in generalised ordered (GO) spaces as follows.

For every uncountable regular cardinal $\kappa$, every GO space has the $B(\kappa)$-property if and only if it has no closed subspace which is homeomorphic to a stationary set in $\kappa$ (with the subspace topology in $\kappa$ ).
\end{abstract}

It is known from [2] that every generalised ordered (GO) space is paracompact if and only if it has no closed subspace which is homeomorphic to a stationary set in some regular uncountable cardinal (for more details, see $[4,3]$ ). In view of this result, I conjectured that for every regular uncountable cardinal $\kappa$, every GO space is $\kappa$-paracompact if and only if it has no closed subspace which is homeomorphic to a stationary set in $\kappa$. But unfortunately, I found a counterexample at once. The ordered space $\omega_{1}$ is not $\omega_{2}$-paracompact, but it has no closed subspace which is homeomorphic to a stationary set in $\omega_{2}$, since the cardinality of $\omega_{1}$ is less than that of $\omega_{2}$. Recently, I proved in [5] that every GO space is paracompact if and only if it has the $\mathcal{B}$-property (defined below) using the result of [2], also that every GO space has the shrinking property (defined below). In this paper, we shall show that for every uncountable regular cardinal $\kappa$, every GO space has the $\mathcal{B}(\kappa)$-property if and only if it has no closed subspace which is homeomorphic to a stationary set in $\kappa$. Furthermore, we shall also clarify the relation between $\kappa$-paracompactness and the $\mathcal{B}(\kappa)$-property in GO spaces.

First we establish our terminology. Let $\kappa$ be an infinite cardinal. A space is said to be $\kappa$-paracompact if every open cover of size $\leqslant \kappa$ has a locally finite open refinement. Note that $\kappa$-paracompactness of a space $X$ is equivalent to the assertion that for every open cover $\left\{U_{\alpha} \mid \alpha<\kappa\right\}$ of $X$ (that is $\bigcup_{\alpha<\kappa} U_{\alpha}=X$ and each $U_{\alpha}$ is open in $X$, but some $U_{\alpha}$ may be empty), there is an open cover $\left\{V_{\alpha} \mid \alpha<\kappa\right\}$ such that $V_{\alpha} \subseteq U_{\alpha}$ for each $\alpha<\kappa$, and for every point $x$ in $X$, there is a neighbourhood $U$ of $x$ such that $\left\{\alpha<\kappa \min U_{\alpha} \cap U \neq \emptyset\right\}$ is finite. We call $\left\{V_{\alpha} \mid \alpha<\kappa\right\}$ a locally finite open refinement of $\left\{U_{\alpha} \mid \alpha<\kappa\right\}$. Let $\mathcal{U}$ be a cover of a space $X$. A cover $\mathcal{F}=\{F(U) \mid U \in \mathcal{U}\}$

Received 17 November 1988

Copyright Clearance Centre, Inc. Serial-fee code: 0004-9729/89 \$A2.00+0.00. 
of $X$ is a shrinking of $\mathcal{U}$ if $\operatorname{cl} F(U) \subseteq U$ for every $U$ in $\mathcal{U}$. A space $X$ has the $\kappa$ shrinking property if every open cover of $X$ of size $\leqslant \kappa$ has a shrinking consisting of open sets (open shrinking in $X$ ). A collection $\left\{U_{\alpha} \mid \alpha<\kappa\right\}$ (indexed by $\kappa$ ) of subsets of a space $X$ is said to be increasing if $U_{\alpha} \subseteq U_{\beta}$ for $\alpha<\beta<\kappa$. A space $X$ has the $\mathcal{B}(\kappa)$-property if every increasing open cover $\left\{U_{\alpha} \mid \alpha<\kappa\right\}$ in $X$ has an increasing open shrinking $\left\{V_{\alpha} \mid \alpha<\kappa\right\}$ in $X$ (that is, $\operatorname{cl} V_{\alpha} \subset U_{\alpha}$ for each $\alpha<\kappa$ ). A space is said to be paracompact (to have the shrinking property, the $\mathcal{B}$-property) if it is $\kappa$ paracompact (has the $\kappa$-shrinking property, the $\mathcal{B}(\kappa)$-property, respectively) for every infinite cardinal $\kappa$. Since in normal spaces, every point finite open cover has an open shrinking, $\kappa$-paracompactness implies the $\kappa$-shrinking property and the $\mathcal{B}(\kappa)$-property. Furthermore $\omega$-paracompactness, the $\omega$-shrinking property and the $\mathcal{B}(\omega)$-property are all equivalent in normal spaces.

A generalised ordered (GO) space is a triple $(X, \mathcal{J},<)$ where $<$ is a linear ordering of the set $X$ and $\mathcal{J}$ is a $T_{1}$-topology which has a base consisting of convex sets, see [2]. Here a subset $C$ of a linearly ordered set $(X,<)$ is convex if $(a, b) \subseteq C$ for every $a, b$ in $C$ with $a<b((a, b)$ denotes the usual open interval with the end points $a, b)$. Note that every open interval $(a, b)$ in GO space $X$ is open with respect to the GO topology $\mathcal{J}$. This means every GO topology is finer than the topology induced by all open intervals, that is, linearly ordered topology. Note that every GO space is hereditary collectionwise normal $([1,6,9])$ and has the shrinking property hereditarily ([5]).

A subset $\mathcal{S}$ of a regular uncountable cardinal $\kappa$ is said to be stationary if $\mathcal{S}$ intersects all closed unbounded (cub) subspaces of $\kappa$ with the order topology. Note that $S \subseteq \kappa$ is stationary if and only if $\mathcal{S}$ does not have the $\mathcal{B}(\kappa)$-property ([5]), where $\kappa$ is regular uncountable. The first purpose of this paper is to enlarge this result for an arbitrary GO space (note that subspaces on linearly ordered topological spaces are GO, in particular such an $S$ is a GO space). It is worth noting that our proofs do not use the complicated notions of cut, gap, $Q$-gap, order compactification,... etcetera. This may enable us (general topologists) to treat covering properties in ordered spaces much more easily without bothering about such complicated notions.

First we establish our main result. The proof is somewhat similar to the proof of 2.1 of $[5]$, but we shall not use the notion of order compactification.

LEMMA 1. Let $X$ be a $G O$ space and $\kappa$ be a regular uncountable cardinal. Assume that $X$ does not have the $\mathcal{B}(\kappa)$-property. Then $X$ contains a closed subspace which is homeomorphic to a stationary set in $\kappa$.

Proof: Assume that a GO space $X$ does not have the $\mathcal{B}(\kappa)$-property. We shall actually construct such a closed subspace. Let $\mathcal{U}=\left\{U_{\alpha}: \alpha<\kappa\right\}$ be an increasing open 
cover of $X$ which does not have an increasing open shrinking in $X$. For every subset $U$ of $X$, define $C(U)$ to be the collection of all maximal convex sets contained in $U$. Note that if $U$ is open, then so is each member of $C(U)$, see [1]. Put $\mathcal{V}=U\left\{C\left(U_{\alpha}\right) \mid \alpha<\kappa\right\}$, and for each $V$ in $\mathcal{V}$, define $\alpha(V)$ to be the least $\alpha<\kappa$ such that $V$ is in $C\left(U_{\alpha}\right)$. For every $V$ and $V^{\prime}$ in $\mathcal{V}$, define $V \simeq V^{\prime}$ if there is a finite subcollection $\left\{V_{0}, \ldots, V_{n}\right\} \subseteq \mathcal{V}$ such that $V=V_{0}, V^{\prime}=V_{n}, V_{i} \cap V_{i+1} \neq \emptyset$ for every $i<n$ (we call such a finite subcollection a "finite chain from $V$ to $V^{\prime \prime}$ ). Then it is easy to show that $\simeq$ is an equivalence relation on $\mathcal{V}$. Let $\left\{\mathcal{V}_{\lambda} \mid \lambda \in \Lambda\right\}$ be the equivalence classes. Then $\left\{U \mathcal{V}_{\lambda} \mid \lambda \in \Lambda\right\}$ decompose $X$ into clopen sets. Since $\mathcal{U}$ does not have an increasing open shrinking in $X$, there is a $\lambda$ in $\Lambda$ such that $\left\{U_{\alpha} \cap\left(U \mathcal{V}_{\lambda}\right) \mid \alpha<\kappa\right\}$ does not have an increasing open shrinking in $U \mathcal{V}_{\lambda}$. Put $Y=U \mathcal{V}_{\lambda}$. First we shall show:

ClaIm 1. For every $y, y^{\prime}$ in $Y$ with $y<y^{\prime}$, there is an $\alpha<\kappa$ such that $\left[y, y^{\prime}\right] \subset$ $U_{\alpha}$.

Proof: Take $V, V^{\prime}$ in $\mathcal{V}_{\lambda}$ such that $y \in V$ and $y^{\prime} \in V^{\prime}$. Then there is a finite chain $\left\{V_{0}, \ldots, V_{n}\right\}$ from $V$ to $V^{\prime}$. We shall show by induction on $n$ that there is a $V^{\prime \prime}$ in $\mathcal{V}$ such that $\left[y, y^{\prime}\right]$ is contained in $V^{\prime \prime}$ (then $\left.\left[y, y^{\prime \prime}\right] \subseteq U_{\alpha\left(V^{\prime \prime}\right)}\right)$. If $n=0$, then there is nothing to prove. Assume it is valid for chains of length $\leqslant(n-1)$. Let $\left\{V_{0}, \ldots, V_{n}\right\}$ be a finite chain from $V$ to $V^{\prime}$ with $y \in V, y^{\prime} \in V^{\prime}$. Take a point $z$ in $V_{n-1} \cap V_{n}$. Then by the inductive assumption, there is a $W$ in $\mathcal{V}_{\lambda}$ such that $[y, z] \subseteq W$. Note that $\left[z, y^{\prime}\right] \subset V_{n}$. There are three cases.

Case 1. $\quad \alpha(W)=\alpha\left(V^{\prime}\right)\left(=\alpha\left(V_{n}\right)\right)=\alpha$.

In this case, since $W$ and $V^{\prime}$ are maximal convex sets contained in $U_{\alpha}$ and $W \cap$ $V^{\prime} \neq \emptyset, W=V^{\prime}$ holds. Then by putting $V^{\prime \prime}=W,\left[y, y^{\prime}\right] \subseteq V^{\prime \prime}$ holds.

Case. $\quad \alpha(W)<\alpha\left(V^{\prime}\right)$.

In this case, since $W \subset U_{\alpha(W)}, V^{\prime} \subset U_{\alpha\left(V^{\prime}\right)}$ and $U_{\alpha(W)} \subset U_{\alpha\left(V^{\prime}\right)}$ hold, we have $W \cup V^{\prime} \subset U_{\alpha\left(V^{\prime}\right)}$. Furthermore, since $W \cup V^{\prime}$ is convex, $W \cup V^{\prime}=V^{\prime}$ holds by the maximality of $V^{\prime}$. Thus by putting $V^{\prime \prime}=V^{\prime}$, we have $\left[y, y^{\prime}\right] \subseteq V^{\prime \prime}$.

Case 3. $\quad \alpha\left(V^{\prime}\right)<\alpha(W)$.

This case is similar to Case 2.

This completes the proof of Claim 1.

To continue the proof, fix a point $c$ in $Y$, and define $Y_{0}=\{y \in Y: y \leqslant c\}$ and $Y_{1}=\{y \in Y: c \leqslant y\}$. We shall prove:

Claim 2. Either $\left\{Y_{0} \cap U_{\alpha} \mid \alpha<\kappa\right\}$ does not have an increasing open shrinking in $Y_{0}$, or $\left\{Y_{1} \cap U_{\alpha} \mid \alpha<\kappa\right\}$ does not have an increasing open shrinking in $Y_{1}$.

Proof: Assume on the contrary that $\left\{Y_{i} \cap U_{\alpha} \mid \alpha<\kappa\right\}$ has an increasing open 
shrinking $\left\{T_{i \alpha} \mid \alpha<\kappa\right\}$ in $Y_{i}$ (that is each $T_{i \alpha}$ is open in $Y_{i}$ and its closure in $Y_{i}$ is contained in $U_{\alpha}$, and $\left\{T_{i \alpha} \mid \alpha<\kappa\right\}$ is an increasing cover of $\left.Y_{i}\right)$ for $i=0,1$. Let $\alpha(i)$ be the least $\alpha<\kappa$ such that $c \in T_{i \alpha}$, for $i=0,1$. Put $\beta=\max \{\alpha(0), \alpha(1)\}$, and define $T_{\alpha}=\emptyset$ for $\alpha<\beta$, and $T_{\alpha}=T_{0 \alpha} \cup T_{1 \alpha}$ (this is open in $Y$, since $X$ is a GO space) for $\alpha \geqslant \beta$. Then $\left\{T_{\alpha} \mid \alpha<\kappa\right\}$ is an increasing open shrinking in $Y$ of $\left\{Y \cap U_{\alpha} \mid \alpha<\kappa\right\}$. But this contradicts our assumption, and completes the proof of Claim 2.

Assume $\mathcal{U}_{1}=\left\{Y_{1} \cap U_{\alpha} \mid \alpha<\kappa\right\}$ does not have an increasing open shrinking in $Y_{1}$ (the remaining case is similar). Next we shall show:

Claim 3. $Y_{1}$ does not have a least upper bound (lub).

Proof: Assume, on the contrary, that $Y_{1}$ has a lub $b$. Then by Claim 1, there is a $\beta<\kappa$ such that $Y_{1}=[c, b] \subseteq U_{\beta}$. By putting $W_{\alpha}=0$ if $\alpha<\beta$, and $W_{\alpha}=Y_{1}$ if $\alpha \geqslant \beta,\left\{W_{\alpha} \mid \alpha<\kappa\right\}$ is an increasing open shrinking in $Y_{1}$ of $U_{1}$. This contradicts our assumption, and completes the proof of Claim 3 .

A subset $A$ of $Y_{1}$ is said to be cofinal in $Y_{1}$ if for every $y$ in $Y_{1}$, there is an $\alpha$ in $A$ such that $y \leqslant \alpha$. Let $\mu$ be the least of $\left\{|A| \mid A\right.$ is cofinal in $\left.Y_{1}\right\}$. Then it is easy to construct a strict increasing cofinal sequence $\left\{\alpha_{\gamma} \gamma<\mu\right\} \subseteq A$ in $Y_{1}$. By the minimality of $\mu$, it is evident that $\mu$ is a regular cardinal. We shall prove:

Claim 4. $\kappa=\mu$.

Proof: For each $\gamma<\mu$, fix an $\alpha(\gamma)<\kappa$ such that $\left[c, \alpha_{\gamma}\right] \subseteq U_{\alpha(\gamma)}$ (by Claim 1).

First assume $\mu<\kappa$. Let $\beta$ be $\sup \{\alpha(\gamma) \mid \gamma<\mu\}$. Then by putting $W_{\alpha}=0$ if $\alpha<\beta$, and $W_{\alpha}=Y_{1}\left(=\cup\left\{\left[c, \alpha_{\gamma}\right] \mid \gamma<\mu\right\} \subseteq U_{\beta} \subseteq U_{\alpha}\right)$ if $\beta \geqslant \alpha,\left\{W_{\alpha} \mid \alpha<\kappa\right\}$ is an increasing open shrinking in $Y_{1}$ of $\mathcal{U}_{1}$. This contradicts to our assumption.

Next assume $\kappa<\mu$. Then there is a $\beta<\kappa$ such that $T=\{\gamma<\mu \mid \alpha(\gamma)=\beta\}$ is stationary in $\mu$. Since $T$ is cofinal in $\mu, Y_{1}=\cup\left\{\left[c, a_{\gamma}\right] \mid \gamma \in T\right\} \subset U_{\beta}$. By putting $W_{\alpha}=0$ if $\alpha<\beta$, and $W_{\alpha}=Y_{1}$ if $\beta \geqslant \alpha .\left\{W_{\alpha} \mid \alpha<\kappa\right\}$ is an increasing open shrinking in $Y_{1}$ of $\mathcal{U}_{1}$. This contradicts our assumption. Thus $\kappa=\mu$ holds.

Put $\mathcal{S}=\left\{\gamma<\kappa \mid\left\{a_{\delta} \mid \delta<\gamma\right\}\right.$ has a lub and $\gamma$ is a limit ordinal $\}$. Define $b_{\gamma}$ to be the lub of $\left\{a_{\delta} \mid \delta<\gamma\right\}$ if $\gamma \in \mathcal{S}$, and $b_{\gamma}=a_{\gamma}$ if $\gamma \in \kappa-\mathcal{S}$. Then it is straightforward to show:

CLAIM 5. $\left\{b_{\gamma} \mid \gamma<\kappa\right\}$ is a strict increasing cofinal sequence in $Y_{1}$ satisfying that, for every ordinal $\gamma<\kappa, \gamma \in \mathcal{S}$ if and only if $\left\{b_{\delta} \mid \delta<\gamma\right\}$ has a lub is $b_{\gamma}$ if it exists) and $\gamma$ is a limit ordinal.

Note that by Claim $5, \mathcal{S}$ is equal to the set $\left\{\gamma<\kappa \mid\left\{b_{\delta} \mid \delta<\gamma\right\}\right.$ has a lub $\left(=b_{\gamma}\right)$ and $\gamma$ is a limit ordinal . 
Claim 6. $\mathcal{S}$ is stationary in $\kappa$.

Proof: Assume indirectly that $\mathcal{S}$ is not stationary. Let $C$ be a cub set consisting of limit ordinals which is disjoint from $S$.

Enumerate $C$ with increasing order, say $\{\gamma(\beta) \mid \beta<\kappa\}$. Define $H_{\beta}$ to be the set $\cup\left\{\left[c, b_{\delta}\right] \mid \delta<\gamma(\beta)\right\}\left(=\cup\left\{\left[c, b_{\delta}\right) \mid \delta<\gamma(\beta)\right\}\right)$ for each $\beta<\kappa$. Since $\gamma(\beta)$ is not in $\mathcal{S}, H_{\beta}$ is closed and open in $Y_{1}$ for each $\beta<\kappa$. Define $K_{\beta}=H_{\beta+1}-H_{\beta}$ for $\beta<\kappa$ with $1 \leqslant \beta$, and $K_{0}=H_{1}$. Since $C$ is cub in $\kappa, Y_{1}$ is the free union (that is, disjoint clopen union) of $\left\{K_{\beta} \mid \beta<\kappa\right\}$. Since $\mathcal{U}_{1}$ has no increasing open shrinking in $Y_{1}$, there is a $\beta<\kappa$ such that $\left\{U_{\alpha} \cap K_{\beta} \mid \alpha<\kappa\right\}$ has no increasing open shrinking in $K_{\beta}$. For each $\delta<\gamma(\beta+1)$, fix an $\alpha(\delta)<\kappa$ such that $\left[c, b_{\delta}\right] \subseteq U_{\alpha(\delta)}$ by Claim 1. By defining $\delta^{\prime}=\sup \{\alpha(\delta) \mid \delta<\gamma(\beta+1)\}, K_{\beta} \subseteq H_{\beta} \subseteq U_{\delta}$, holds. Previously, by putting $W_{\alpha}=0$ if $\alpha<\delta^{\prime}$, and $W_{\alpha}=K_{\beta}$ if $\alpha \geqslant \delta^{\prime},\left\{W_{\alpha} \mid \alpha<\kappa\right\}$ is an increasing open shrinking in $K_{\beta}$ of $\left\{U_{\alpha} \cap K_{\beta} \mid \alpha<\kappa\right\}$. This is a contradiction. Thus $\mathcal{S}$ is stationary. This completes the proof of Claim 6.

Finally, define $Z=\left\{b_{\gamma} \mid \gamma \in \mathcal{S}\right\}, h(\gamma)=b_{\gamma}$ for every $\gamma$ in $\mathcal{S}$. Then it is straightforward to show that $Z$ is closed in $X$ and $h$ is a homeomorphism of $\mathcal{S}$ onto $Z$. Thus the proof of the lemma is complete.

ThEOREM 2. Let $X$ be a GO space, and $\kappa$ be a regular uncountable cardinal. Then $X$ does not have the $\mathcal{B}(\kappa)$-property if and only if $X$ has a closed subspace which is homeomorphic to a stationary set in $\kappa$.

Proof: One direction is Lemma 1 . Since every stationary set in $\kappa$ does not have the $\mathcal{B}(\kappa)$-property $([5,3.11])$ and the $\mathcal{B}(\kappa)$-property is a closed hereditary property, the other direction also holds.

Since $\mathcal{B}(\kappa)$-property is equivalent to $\mathcal{B}(\mathrm{c}\lceil\kappa)$-property (the proof is easy), Theorem 2 can be restated as follows (here $\mathrm{cf} \kappa$ denotes the cofinality of $\kappa$ ). Note that every G0 space is hereditary countably paracompact (thus has the $\mathcal{B}(\omega)$-property hereditarily), thus every GO space has the $\mathcal{B}(\kappa)$-property hereditarily whenever $\kappa$ is a cardinal of countable cofinality.

THEOREM 2'. Let $X$ be a $G O$ space, and $\kappa$ be a cardinal of uncountable cofinality. Then $X$ does not have the $\mathcal{B}(\kappa)$-property if and only if $X$ has a closed subspace which is homeomorphic to a stationary set in $\mathrm{cf} \kappa$.

To investigate relations between $\kappa$-paracompactness and the $\mathcal{B}(\kappa)$-property in GO spaces, we shall establish:

LEMMA 3. Let $X$ be a $G O$ space, and $\kappa$ be an uncountable regular cardinal. Assume that there is an increasing open cover $\mathcal{U}=\left\{U_{\alpha}: \alpha<\kappa\right\}$ which does not 
have a locally finite open refinement. Then there is a closed subspace of $X$ which is homeomorphic to a stationary set in $\kappa$.

Proof: The proof of this lemma is completely analogous to that of Lemma 1, by replacing "increasing open cover" (in the proof of Lemma 1) by "locally finite open refinement" (in the proof of this lemma). But we should note the proof of Claim 2. Claim 2 of Lemna 3 is stated as follows.

Claim 2. Either $\left\{Y_{0} \cap U_{\alpha}: \alpha<\kappa\right\}$ does not have a locally finite open refinement in $Y_{0}$, or $\left\{Y_{1} \cap U_{\alpha}: \alpha<\kappa\right\}$ does not have a locally finite open refinement in $Y_{1}$.

Proof: Assume on the contrary that $\left\{Y_{i} \cap U_{\alpha} \mid \alpha<\kappa\right\}$ has a locally finite open refinement $\left\{T_{i \alpha} \mid \alpha<\kappa\right\}$ in $Y_{i}$ for $i=0,1$. Fix an $\alpha_{0}<\kappa$ with $c \in U_{\alpha 0}$. Then $\left\{T_{0 \alpha}-\{c\} \mid \alpha<\kappa\right\} \cup\left\{T_{1 \alpha}-\{c\} \mid \alpha<\kappa\right\} \cup\left\{U_{\alpha 0}\right\}$ is a locally finite open refinement in $Y$ of $\left\{Y \cap U_{\alpha} \mid \alpha<\kappa\right\}$, since $X$ is GO. Thus as in the proof of Claim 2 of Lemma 1, we obtain a contradiction.

The remaining parts are all similar. This completes the proof of the Lemma.

Tireorem 4. Let $X$ be a $G O$ space, and $\kappa$ be an uncountable cardinal. Then $X$ is $\kappa$-paracompact if and only if $X$ has the $\mathcal{B}(\lambda)$-property for every regular uncountable cardinal $\lambda$ with $\lambda \leqslant \kappa$.

Proof: One direction is obvious. To show the other direction, assume indirectly that $\kappa$ is the least cardinal such that there is a GO space $X$ which is not $\kappa$-paracompact, but has the $\mathcal{B}(\lambda)$-property for every regular uncountable cardinal $\lambda$ with $\lambda \leqslant \kappa$. By the minimality of $\kappa$, such an $X$ is $\kappa^{\prime}$-paracompact for every infinite cardinal $\kappa^{\prime}$ with $\kappa^{\prime}<\kappa$. Since $X$ is not $\kappa$-paracompact, take an open cover $U \mathcal{V}$ of size $\kappa$ which does not have a locally finite open refinement in $X$, say $\cup \mathcal{V}=\left\{W_{\alpha} \mid \alpha<\kappa\right\}$. Note that $\omega<\kappa$, since GO spaces are countably paracompact. First we shall show that $\kappa$ is regular. Assume on the contrary that $\kappa$ is singular. Fix a cofinal strict increasing sequence $\{\kappa(\gamma) \mid \gamma<\operatorname{cf} \kappa\}$ in $\kappa$. By the $\mathcal{B}(\mathrm{cf} \kappa)$-property, take an increasing open shrinking $\left\{W_{\gamma}^{\prime} \mid \gamma<\operatorname{cf} \kappa\right\}$ of the increasing open cover $\left\{U_{\alpha<\kappa(\gamma)} W_{\alpha} \mid \gamma<\operatorname{cf} \kappa\right\}$. By cf $\kappa$-paracompactness, take a locally finite open refinement $\left\{W_{\gamma}^{\prime \prime} \mid \gamma<\operatorname{cf} \kappa\right\}$ of $\left\{W_{\gamma}^{\prime} \mid \gamma<\operatorname{cf} \kappa\right\}$. Then $\left\{W_{\alpha} \mid \alpha<\kappa(\gamma)\right\}$ covers $\mathrm{cl} W_{\gamma}^{\prime \prime}$ for each $\gamma<\mathrm{cf} \kappa$. By $\kappa(\gamma)$-paracompactness, take a locally finite open refinement (in $\mathrm{cl} W_{\gamma}^{\prime \prime}$ ) $\left\{W_{\alpha \gamma} \mid \alpha<\kappa(\gamma)\right\}$ of $\left\{W_{\alpha} \cap \mathrm{cl} W_{\gamma}^{\prime \prime} \mid \alpha<\kappa(\gamma)\right\}$ for each $\gamma<\operatorname{cf} \kappa$. Then it is straightforward to show that $U\left\{\left\{W_{\alpha \gamma} \cap W_{\gamma}^{\prime \prime} \mid \alpha<\kappa(\gamma)\right\} \mid \gamma<\operatorname{cf} \kappa\right\}$ is a locally finite open refinement of $\cup \mathcal{V}$. This is a contradiction. Thus $\kappa$ is a regular uncountable cardinal.

Next by the $\mathcal{B}(\kappa)$-property of $X$, take an increasing open shrinking $U=\left\{U_{\alpha} \mid\right.$ $\alpha<\kappa\}$ of $\left\{U_{\beta<\alpha} W_{\beta} \mid \alpha<\kappa\right\}$. We shall show that the increasing open cover $U$ has no locally finite open refinement. To show this, assume indirectly that $U$ has a 
locally finite open refinement $\left\{\begin{array}{l}\prime \\ \alpha\end{array} \alpha<\kappa\right\}$. Since $U_{\alpha}^{\prime} \subseteq U_{\alpha} \subseteq \operatorname{cl} U_{\alpha} \subseteq U_{\beta<\alpha} W_{\beta}$, take a locally finite open refinement (in $\operatorname{cl} U_{\alpha}^{\prime}$ ) $\left\{W_{\beta \alpha} \mid \beta<\alpha\right\}$ of $\left\{\mathrm{cl} U_{\alpha}^{\prime} \cap W_{\beta} \mid \beta<\alpha\right\}$ by $|\alpha|$-paracompactness of $X$ for each $\alpha<\kappa$. Then $\cup\left\{\left\{W_{\beta \alpha} \cap U_{\alpha}^{\prime} \mid \beta<\alpha\right\} \mid \alpha<\kappa\right\}$ is a locally finite open refinement (in $X$ ) of $\cup \mathcal{V}$. But this is a contradiction. Therefore $\mathcal{U}$ is an increasing open cover which does not have a locally finite open refinement. It follows from Lemma 3 that $X$ has a closed subspace which is homeomorphic to a stationary set in $\kappa$. This implies $X$ does not have the $\mathcal{B}(\kappa)$-property by Theory 2. This contradicts our assumption. The proof is complete.

Remark. The Navy space is not paracompact ([7]), but has the $\mathcal{B}$-property $([8])$. Thus we can not omit the condition GO from Theorem 4.

The result of [2] is now clear by Theorem 2 and 4 .

Corollary 5. ([5]) Let $X$ be a GO space. Then $X$ is paracompact if and only if $X$ does not have a closed subspace which is homeomorphic to a stationary set in some regular uncountable cardinal.

\section{REFERENCES}

[1] R. Engelking, General Topology (PWN-Polish Scientific Publisherers, Warszawa, 1977).

[2] R. Engelking and D.J. Lutzer, 'Paracompactness in ordered spaces', Fund. Math. O4 (1977), $49-58$.

[3] V.V. Fedorcuk, 'Some questions in the theory of ordered spaces', Siberian Math. J. 10 (1969), 124-132.

[4] L. Gillman and M. Henriksen, 'Concerning rings of continuous functions', Trans. Amer. Math. Soc. 77 (1954), 340-362.

[6] N. Kemoto, 'The shrinking property and the B-property in ordered spaces', Fund. Math. (to 8ppear).

[6] M.J. Mansfield, 'Some generalizations of full normality', Trans. Amer. Math. Soc. 86 (1957), 443-449.

[7] K. Navy, 'ParaLindelöfness and paracompactness'. (Thesis, University of Wisconsin, Madison, 1981).

[8] M.E. Rudin, 'Yasui's questions', Questions Answers Gen. Topology 1 (1983), 122-127.

[8] L.A. Steen, 'A direct proof that a linearly ordered space is hereditarily collectionwise normal', Proc. Amer. Math. Soc. 24 (1970), 727-728.

Department of Mathematics

Faculty of Education

Oita University

Dannoharu Oita 870-11

Japan 